\title{
Note
}

\section{Re-examination of selection index for desired gains}

\author{
Y. ITOH and Y. YAMADA \\ Department of Animal Science, College of Agriculture, \\ Kyoto University, Kyoto 606, Japan
}

\begin{abstract}
Summary
The method of index selection for desired genetic changes derived by Brascamp (1979) was re-examined and we found that his method gave the equivalent solutions to that of YAMADA et al. (1975). Brascamp's method is more complicated and has no advantage compared to the method of YAMADA et al.
\end{abstract}

Key words : Selection index, desired gains, constrained selection index.

\section{Résumé}

Nouvel examen des index de sélection pour des gains génétiques espérés

Nous avons réexaminé la méthode de sélection par index pour des gains génétiques espérés proposée par Brascamp (1979) et nous trouvons que sa méthode fournit des solutions équivalentes à celles de YAMADA et al. (1975). La méthode de Brascamp est plus compliquée et ne présente pas d'avantage par rapport à celle de YAMADA et al.

Mots clés : Index de sélection, gains espérés, index de sélection sous contrainte.

A selection index for desired genetic changes was derived by PeSEK \& BaKer (1969). They restricted themselves to the situations where the traits in the index are exactly the same as those in the aggregate genotype. YAmADA et al. (1975) gave a more general solution. Their method is available even in situations where the index contains traits not included in the aggregate genotype. Similar results have been obtained by Harville (1975), Rouvier (1977), Essl (1981) and TAllis (1985). The most different point of YAMADA et al. (1975) from the others was that they did not assume any economic weight nor underlying aggregate genotypic value.

Brascamp (1979) discussed another solution of this problem and a detailed derivation of his method was written in his review paper (BRASCAMP, 1984). His method 
appears quite different from that of YAMADA et al. (1975), but we found that they were exactly the same. Because the method of YAMADA et al. is simpler than that of Brascamp, we can say that the former is preferable to the latter.

The objective of this note is to prove their equivalence algebraically.

To describe the selection indices we use the following notations. $b$ is an $n \times 1$ vector of weighting factors. $\mathbf{p}$ is an $n \times 1$ vector of phenotypic values of individuals as deviations from their relevant means. $\mathbf{g}$ is an $m \times 1$ vector of additive genetic merits. $\mathbf{d}$ is an $m \times 1$ vector of desired relative genetic changes. The variance covariance matrix of $\mathbf{p}$ is denoted as $\mathbf{P}$ with order $\mathbf{n} \times \mathbf{n}$. The covariance matrix between $\mathbf{p}$ and $\mathbf{g}$ is denoted as $\mathbf{G}$ with order $n \times m$, i.e. $\operatorname{Cov}(\mathbf{p}, \mathbf{g})=\mathbf{G}$. We do not assume any economic weight, nor aggregate genotypic value.

First we describe the selection index of Yamade et al. (1975). The expected gains after applying the selection index $I=\mathbf{b}^{\prime} \mathbf{p}$ in one generation are :

$$
\mathrm{E}(\Delta \mathbf{g})=i \mathbf{G}^{\prime} \mathbf{b} / \sigma_{I}
$$

where $i$ is the intensity of selection and $\sigma_{I}$ is the standard deviation of the index, i.e. $\sigma_{I}=\sqrt{\mathbf{b}^{\prime} \mathbf{P b}}$. Because $\mathrm{E}(\Delta \mathbf{g})$ is proportional to $i$ and $\sigma_{l}$, it is sufficient to solve $\mathbf{b}$ such that :

$$
\mathbf{G}^{\prime} \mathbf{b}=\mathbf{d}
$$

then $\mathrm{E}(\Delta \mathbf{g})=i \mathbf{d} / \sigma_{I}$. Now suppose $n>m$ and $\mathbf{G}$ has full column rank. If $n<m$ or $\mathbf{G}$ is not of full column rank, equations (1) may be inconsistent and have no solution in general. If these equations are consistent, they have solutions, but no unique solution exists. Now $\mathrm{E} \Delta \mathrm{g}$ is inversely proportional to $\sigma_{l}$, so that the best choice among all solutions is b causing $\sigma_{l}$ to be minimum subject to the constraints (1), because then $\mathrm{E}(\Delta \mathrm{g})$ is maximum. Such a solution can be found by putting partial derivatives of :

$$
\frac{1}{2} \mathbf{b}^{\prime} \mathbf{P b}+\boldsymbol{\lambda}^{\prime}\left(\mathbf{G}^{\prime} \mathbf{b}-\mathbf{d}\right)
$$

with respect to $\mathbf{b}$ and $\boldsymbol{\lambda}$ to zeros, where $\boldsymbol{\lambda}$ is an $m \times 1$ vector of Lagrange multipliers. Then we get the equations :

$$
\left[\begin{array}{ll}
\mathbf{P} & \mathbf{G} \\
\mathbf{G}^{\prime} & \mathbf{0}
\end{array}\right]\left[\begin{array}{l}
\mathbf{b} \\
\boldsymbol{\lambda}
\end{array}\right]=\left[\begin{array}{l}
\mathbf{0} \\
\mathbf{d}
\end{array}\right]
$$

and solving these equations as to $\mathbf{b}$, we finally get :

$$
\mathbf{b}=\mathbf{P}^{-1} \mathbf{G}\left(\mathbf{G}^{\prime} \mathbf{P}^{-1} \mathbf{G}\right)^{-1} \mathbf{d}
$$

which is the selection index derived by Yamada et al. (1975).

This result is also derived easily from the fact that $\mathbf{P}^{-1} \mathbf{G}\left(\mathbf{G}^{\prime} \mathbf{P}^{-1} \mathbf{G}\right)^{-1}$ is a minimum norm generalized inverse of $\mathbf{G}^{\prime}$.

Brascamp (1979) modified the equations (1) as :

$$
\mathbf{G G}^{\prime} \mathbf{b}=\mathbf{G d}
$$


by pre-multiplying G. Because GG' $^{\prime}$ is singular, no unique solution for b exists, and all solutions can be denoted by :

$$
\mathbf{b}=\left(\mathbf{G G}^{\prime}\right)^{-\mathbf{G d}}+\left[\left(\mathbf{G G}^{\prime}\right)^{-} \mathbf{G} \mathbf{G}^{\prime}-\mathbf{I}\right] \mathbf{z}=\mathbf{A d}+\mathbf{B z}
$$

where $\mathbf{A}=\left(\mathbf{G G}^{\prime}\right)^{-} \mathbf{G}, \mathbf{B}=\left(\mathbf{G G}^{\prime}\right)^{-} \mathbf{G G}^{\prime}-\mathbf{I},\left(\mathbf{G G}^{\prime}\right)^{-}$is a generalized inverse of $\mathbf{G G}^{\prime}$ and $\mathbf{z}$ is an arbitrary $n \times 1$ vector. The best choice among all solutions is $\mathbf{b}$ causing the variance of the index to be minimum. It can be shown that $z=-\left(B^{\prime} \mathbf{P B}\right)^{-B^{\prime}}$ PAd minimizes $\mathbf{b}^{\prime} \mathbf{P b}$. Substituting this $\mathbf{z}$ into (5), we get :

$$
\mathbf{b}=\left[\mathbf{I}-\mathbf{B}\left(\mathbf{B}^{\prime} \mathbf{P B}\right)^{-} \mathbf{B}^{\prime} \mathbf{P}\right] \mathbf{A d}
$$
(1984).

The resulting solution is invariant to the choice of $\left(\mathbf{B}^{\prime} \mathbf{P B}\right)^{-}$as proved by BrasCAMP

The method of Brascamp looks quite reasonable, but it has an improper point shown in the following. The equations (4) look like normal equations used in the least squares procedure. That is, it seems as if he applied the least squares procedure to (1) and get the normal equations (4). The equations (1), however, are consistent, so they hold good without error and it is obvious that their solutions are exactly the same as those of the equations (4). Further both of the method given by Yamada et al. and BrasCamp adopt $b$ which minimizes the variance of the index. These facts can make us understand intuitively the equivalence of these 2 methods.

Now we can prove the equivalence of these methods algebraically. We must use the following lemma.

Lemma 1. Let $\mathbf{X}_{p \times r}$ and $\mathbf{K}_{p \times(p-q)}$ be of rank $q$ and $(p-q)$ such that $\mathbf{K}^{\prime} \mathbf{X}=\mathbf{0}$. Then if $\mathbf{V}_{p \times p}$ is a symmetric positive definite matrix, then :

$$
\mathbf{V}^{-1}-\mathbf{V}^{-1} \mathbf{X}\left(\mathbf{X}^{\prime} \mathbf{V}^{-1} \mathbf{X}\right)^{-} \mathbf{X}^{\prime} \mathbf{V}^{-1}=\mathbf{K}\left(\mathbf{K}^{\prime} \mathbf{V K}\right)^{-1} \mathbf{K}^{\prime}
$$

The proof of this lemma is indicated in an appendix.

B has order $n \times n$ and rank $(n-m)$, $\mathbf{G}$ has order $n \times m$ and rank $m, \mathbf{P}$ is symmetric positive definite, and further:

$$
\mathbf{G}^{\prime} \mathbf{B}=\mathbf{G}^{\prime}\left(\mathbf{G G}^{\prime}\right)^{-} \mathbf{G G}^{\prime}-\mathbf{G}^{\prime}=\mathbf{G}^{\prime}-\mathbf{G}^{\prime}=0,
$$

so that, using lemma 1 , it can be shown that :

$$
\mathbf{P}-\mathbf{P B}\left(\mathbf{B}^{\prime} \mathbf{P B}\right)^{-} \mathbf{B}^{\prime} \mathbf{P}=\mathbf{G}\left(\mathbf{G}^{\prime} \mathbf{P}^{-1} \mathbf{G}\right)^{-1} \mathbf{G}^{\prime}
$$

Using this, Brascamp's selection index can be rewritten as :

$$
\begin{aligned}
\mathbf{b} & =\left[\mathbf{I}-\mathbf{B}\left(\mathbf{B}^{\prime} \mathbf{P B}\right)^{-} \mathbf{B}^{\prime} \mathbf{P}\right] \mathbf{A d} \\
& =\mathbf{P}^{-1}\left[\mathbf{P}-\mathbf{P B}\left(\mathbf{B}^{\prime} \mathbf{P B}\right)^{-} \mathbf{B}^{\prime} \mathbf{P}\right] \mathbf{A d} \\
& =\mathbf{P}^{-1} \mathbf{G}\left(\mathbf{G}^{\prime} \mathbf{P}^{-1} \mathbf{G}\right)^{-1} \mathbf{G}^{\prime} \mathbf{A d}
\end{aligned}
$$

Because $\mathbf{G}$ has full column rank, it can be partitioned as :

$$
\mathbf{G}=\left[\begin{array}{l}
\mathbf{G}_{1} \\
\mathbf{G}_{2}
\end{array}\right]
$$


where $\mathbf{G}_{1}$ is an $m \times m$ non-singular matrix and $\mathbf{G}_{2}$ is an $(n-m) \times m$ matrix. We use the following generalized inverse of $\mathbf{G G}^{\prime}$ for simplification.

$$
\left(\mathbf{G G}^{\prime}\right)^{-}=\left[\begin{array}{cc}
\left(\mathbf{G}_{1} \mathbf{G}_{1}\right)^{-1} & \mathbf{0} \\
\mathbf{0} & \mathbf{0}
\end{array}\right]
$$

Then we get :

$$
\mathbf{G}^{\prime} \mathbf{A}=\mathbf{G}^{\prime}\left(\mathbf{G G}^{\prime}\right)^{-} \mathbf{G}=\mathbf{G}_{1}^{\prime}\left(\mathbf{G}_{1} \mathbf{G}_{1}\right)^{-1} \mathbf{G}_{1}=\mathbf{I}
$$

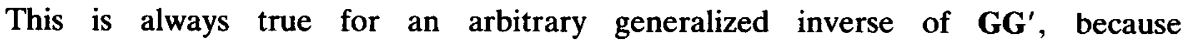
$\mathbf{G}^{\prime}\left(\mathbf{G G}^{\prime}\right)^{-} \mathbf{G}$ is invariant to the choice of the generalized inverse as well known. Using this result, (7) becomes :

$$
\begin{aligned}
\mathbf{b} & =\mathbf{P}^{-1} \mathbf{G}\left(\mathbf{G}^{\prime} \mathbf{P}^{-1} \mathbf{G}\right)^{-1} \mathbf{G}^{\prime} \mathbf{A d} \\
& =\mathbf{P}^{-1} \mathbf{G}\left(\mathbf{G}^{\prime} \mathbf{P}^{-1} \mathbf{G}\right)^{-1} \mathbf{d}
\end{aligned}
$$

This formula is exactly the selection index of Yamada et al. represented by (3).

As mentioned above, these 2 methods of YAMADA $e t$ al. and Brascamp give equivalent solutions. Furthermore, BrascAMP's method is more complicated than that of YAMADA et al. Therefore, BRASCAMP's method has no advantage compared to that of YAMADA et al.

\section{Numerical Example}

We use the same example used by Brascamp (1979) as follows.

$$
\begin{aligned}
& \mathbf{P}=\left[\begin{array}{rrr}
100.00 & 30.00 & 15.00 \\
& 36.00 & 18.00 \\
& & 25.00
\end{array}\right], \mathbf{G}=\left[\begin{array}{rr}
25.00 & 9.00 \\
9.00 & 12.96 \\
6.00 & 8.64
\end{array}\right], \\
& \mathbf{d}=\left[\begin{array}{l}
1 \\
2
\end{array}\right]
\end{aligned}
$$

We can use the 3 different formulae (2), (3) and (6) to compute the selection index, but whichever formula we may use, we get the identical solution :

$$
\mathbf{b}=\left[\begin{array}{r}
-0.020741 \\
0.133091 \\
0.053450
\end{array}\right]
$$


Then the variance of the selection index is :

$$
\sigma_{I}^{2}=b^{\prime} \mathbf{P b}=0.809327
$$

The expected genetic gains in one generation of selection are :

$$
\mathrm{E}(\Delta \mathbf{g})=\frac{i \mathrm{~d}}{\sigma_{I}}=i\left[\begin{array}{l}
1.111573 \\
2.223146
\end{array}\right]
$$

\section{Acknowledgement}

The authors thank the referees for their usefull suggestions. This study was supported in part by Grants-in-aid for Scientific Research No. 61304029 from the Ministry of Education, Science and Culture.

Received November 19, 1985.

Accepted May 21, 1986.

\section{References}

Brascamp E.W., 1979. Selection index for desired gains. 30th Annual Meeting of the European Association of Animal Production, Harrogate, England, G6.5.

Brascamp E.W., 1984. Selection indices with constraints. Anim. Breed. Abstr., 53, 645-654.

EsSL A., 1981. Index selection with proportionality restriction: Another viewpoint. Z. Tierz. Züchtungsbiol., 98, 125-131.

Harville D.A., 1975. Index selection with proportionality constraints. Biometrics, 31, 223-225.

Khatri C.G., 1966. A note on a MANOVA model applied to problems in growth curve. Ann. Inst. Stat. Math., 18, 75-86.

Pesek J., Baker R.J., 1969. Desired improvement in relation to selection indices. Can. J. Plant Sci., 49, 803-804.

Rouvier R., 1977. Mise au point sur le modèle classique d'estimation de la valeur génétique. Ann. Génét. Sél. Anim., 9 (1), 17-26.

SEARLE S.R., 1979. Notes on variance component estimation: a detailed account of maximum likelihood and kindred methodology. Paper BU-673-M in Biometrics Unit, Cornell University.

TAllis G.M., 1985. Constrained selection. Jpn. J. Genet., 60, 151-155.

Yamada Y., Yokouchi K., Nishida A., 1975. Selection index when genetic gains of individual traits are of primary concern. Jpn. J. Genet., 50, 33-41.

\section{Appendix}

\section{Proof of lemma 1}

Let us partition $\mathbf{X}$ as $\mathbf{X}=[\mathbf{W}: \mathbf{W F}]$, where $\mathbf{W}$ is of order $\mathrm{p} \times \mathrm{q}$ and full column rank and $\mathrm{F}$ is of order $q \times(r-q)$. Because $\mathbf{K}^{\prime} \mathbf{X}=\mathbf{0}, \mathbf{W}$ also satisfies the condition $\mathbf{K}^{\prime} \mathbf{W}=\mathbf{0}$. Because $\mathbf{V}$ is symmetric positive definite, there exists a non-singular matrix 
$\mathbf{T}_{p \times p}$ such that $\mathbf{V}=\mathbf{T} \mathbf{T}^{\prime}$. Similarly let $\left(\mathbf{W}^{\prime} \mathbf{V}^{-1} \mathbf{W}\right)^{-1}=\mathbf{Q} \mathbf{Q}^{\prime}$ and $\left(\mathbf{K}^{\prime} \mathbf{V K}\right)^{-1}=\mathbf{R} \mathbf{R}^{\prime}$ where $\mathbf{Q}_{q \times q}$ and $\mathbf{R}_{(p-q) \times(p-q)}$ are non-singular matrices. We define $S_{p \times p}$ as $\mathbf{S}=\left[\mathbf{T}^{-1} \mathbf{W} \mathbf{Q}: \mathbf{T}^{\prime} \mathbf{K} \mathbf{R}\right]$. Then $\mathbf{S}$ has full rank and $\mathbf{S}^{\prime} \mathbf{S}=\mathbf{I}_{p}$, so that $\mathbf{S}$ is an orthogonal matrix. Therefore :

$$
\mathbf{I}_{p}=\mathbf{S S}^{\prime}=\mathbf{T}^{-1} \mathbf{W Q Q} \mathbf{Q}^{\prime} \mathbf{W}^{\prime} \mathbf{T}^{\prime-1}+\mathbf{T}^{\prime} \mathbf{K R R} \mathbf{R}^{\prime} \mathbf{T},
$$

then :

$$
\mathbf{I}-\mathbf{T}^{-1} \mathbf{W}\left(\mathbf{W}^{\prime} \mathbf{V}^{-1} \mathbf{W}\right)^{-1} \mathbf{W}^{\prime} \mathbf{T}^{\prime-1}=\mathbf{T}^{\prime} \mathbf{K}\left(\mathbf{K}^{\prime} \mathbf{V K}\right)^{-1} \mathbf{K}^{\prime} \mathbf{T}
$$

Pre-multiplying $\mathbf{T}^{\prime-1}$ and post-multiplying $\mathbf{T}^{-1}$, we get :

$$
\mathbf{V}^{-1}-\mathbf{V}^{-1} \mathbf{W}\left(\mathbf{W}^{\prime} \mathbf{V}^{-1} \mathbf{W}\right)^{-1} \mathbf{W}^{\prime} \mathbf{V}^{-1}=\mathbf{K}\left(\mathbf{K}^{\prime} \mathbf{V K}\right)^{-1} \mathbf{K}^{\prime}
$$

This is the result derived by KHATRI (1966). (In his original paper, the definition of the matrix $\mathbf{S}$ was $\mathbf{S}=\left[\mathbf{T}^{-1} \mathbf{W Q}: \mathbf{T K R}\right]$. However, if we use it, we can not get the result. It should be $\left.\mathbf{S}=\left[\mathbf{T}^{-1} \mathbf{W Q}: \mathbf{T}^{\prime} \mathbf{K R}\right]\right)$. On the other hand,

$$
\begin{aligned}
\mathbf{X}\left(\mathbf{X}^{\prime} \mathbf{V}^{-1} \mathbf{X}\right)^{-} \mathbf{X}^{\prime} & =[\mathbf{W} \vdots \mathbf{W F}]\left[\begin{array}{cc}
\mathbf{W}^{\prime} \mathbf{V}^{-1} \mathbf{W} & \vdots \mathbf{W}^{\prime} \mathbf{V}^{-1} \mathbf{W F} \\
\mathbf{F}^{\prime} \mathbf{W}^{\prime} \mathbf{V}^{-1} \mathbf{W} & \vdots \mathbf{F}^{\prime} \mathbf{W}^{\prime} \mathbf{V}^{-1} \mathbf{W F}
\end{array}\right]-\left[\begin{array}{l}
\mathbf{W}^{\prime} \\
\mathbf{F}^{\prime} \mathbf{W}^{\prime}
\end{array}\right] \\
& =[\mathbf{W} \vdots \mathbf{W F}]\left[\begin{array}{cc}
\left(\mathbf{W}^{\prime} \mathbf{V}^{-1} \mathbf{W}\right)^{-1} & \vdots \mathbf{0} \\
0 & \vdots \mathbf{0}
\end{array}\right]\left[\begin{array}{l}
\mathbf{W}^{\prime} \\
\mathbf{F}^{\prime} \mathbf{W}^{\prime}
\end{array}\right] \\
& =\mathbf{W}\left(\mathbf{W}^{\prime} \mathbf{V}^{-1} \mathbf{W}\right)^{-1} \mathbf{W}^{\prime} .
\end{aligned}
$$

This is always true for any generalized inverse of $\mathbf{X}^{\prime} \mathbf{V}^{-1} \mathbf{X}$, because $\mathbf{X}\left(\mathbf{X}^{\prime} \mathbf{V}^{-1} \mathbf{X}\right)^{-} \mathbf{X}^{\prime}$ is invariant to the choice of the generalized inverse. Substituting (A2) into (A1), we get :

$$
\mathbf{V}^{-1}-\mathbf{V}^{-1} \mathbf{X}\left(\mathbf{X}^{\prime} \mathbf{V}^{-1} \mathbf{X}\right)^{-} \mathbf{X}^{\prime} \mathbf{V}^{-1}=\mathbf{K}\left(\mathbf{K}^{\prime} \mathbf{V K}\right)^{-1} \mathbf{K}^{\prime}
$$

For more detailed discussions, see SEARLE (1979). 